\title{
Cloud Computing and Healthcare Services
}

\section{Aziz HA ${ }^{1 *}$ and Guled $A^{2}$}

${ }^{1}$ College of Arts and Sciences, Qatar University, Qatar

${ }^{2}$ Qatar University, Doha, Qatar

\begin{abstract}
There is a significant volume of healthcare data generated daily. The data are important and vital for decision making and delivering the best care for patients. Cloud computing is a cost effective method that facilitates real-time data collection, data storage and exchange between healthcare organizations. Cloud infrastructure is characterized with a high throughput and a high volume storage; two important factors for efficient data analysis of large patients' population. Security and privacy are of the major concerns for using cloud-based healthcare services. Healthcare organization should have electronic medical records in order to use the cloud infrastructure. In order to cope with the rapid advancements in information technology and the utilization of cloud based services, efforts should be dedicated to move healthcare data form the traditional paper based to the electronic format. Then, regional legislation and policies should be enacted to regulate and control the usage of healthcare data.
\end{abstract}

Keywords: Cloud computing; Cloud system; Healthcare organizations; Electronic health records; Security and privacy; Body sensors

\section{Introduction}

Utilization of information technology in the healthcare sector is significantly underutilized, particularly in areas of operational efficiency. Most healthcare practitioners and institutions still rely on paper medical records, thus limiting collaboration and coordination between patients and physicians. Any modernization efforts in healthcare must include cloud computing as a centre piece for this transformation. According to the National Institute of Standards and Technology (NIST), cloud computing can serve as a model that permits a set of configurable computing resources in sharing convenient and on-demand network access that are not usually provided in regular healthcare environments. Cloud computing has both the broad network access and resource pooling to support big data sets from electronic healthcare records (EHR). Both features facilitate the usage of computing resources by multiple consumers through different electronic devices such as mobile phones and tablets, providing more timely access to critical medical data. In addition, a cloud computing system is a measured service that has the ability to analyse and control resource usage in regards to the type of service provided e.g., storage and processing. In order for cloud computing to provide clients with the desirable services, it should be built on an infrastructure that facilitates the expected functions. The infrastructure is a combination of hardware and software resources, also referred to physical and abstraction layers, respectively. The physical layer includes the server, storage and network components, whereas the abstraction layer consists of the software installed to carry out the service. When it comes to consumers and their role in the cloud system, they don't need to manage the infrastructure itself; however they do have the privilege of controlling applications and configuration settings. Consequently, the cloud infrastructure can be provided for organization and their multiple consumers, also for specific community or it could be for the general public [1]. In healthcare organizations, there are enormous amounts of data collected and stored daily. These data are important and vital for decision making and for delivering the appropriate treatment. Healthcare organizations need to have EHRs to be able to exchange data using cloud computing systems where patients' data are stored, retrieved and shared with other healthcare providers. Since the number of the healthcare data is continuously increasing, it will eventually affect the storage capacity of healthcare data servers and cause slowness in data retrieval. Cloud computing is the solution for the "big data" challenge [2]. It provides unlimited storage resources and facilitates the process of sharing patients' data between healthcare organizations [3].

\section{Cloud computing and healthcare organizations}

The dramatic increase in average life expectancy led to faster population aging. This created a growing demand for additional resources and a diverse medical care. Innovative and cost-effective methods are required to assist healthcare providers find more productive ways in addressing this global challenge. Cloud computing can offer practical solutions as in the new clinical information management system called "Collaborative Care Solution" that was developed in November 2010 by IBM and Active Health Management. The system was based on cloud computing with a purpose to help medical and healthcare staff to easily access healthcare data and information from different sources such as EHR. On the other side, it was beneficial for patients who were suffering from chronic conditions to connect with their physicians and follow up their prescribed medications. Moreover, healthcare institutions found the system to be extremely cost effective. Some of these benefits were eliminating the need to update the information technology systems when there was a change in the clinical guidelines or reporting requirements.

Likewise, management of data was more efficient in regards to the growing numbers of patients' data and information through electronic and personal health records. This could be viewed from the perspective of data storage and the number of servers needed to cope up with theses enormous amounts of data. What facilitates the function of cloud computing is the usage of smart phones and tablets that support medical staff and patients to access healthcare services. This movement

*Corresponding author: Hassan A Aziz, PhD, MLS(ASCP)cm, Associate Dean for Academic Affairs, College of Arts and Sciences, Qatar University, P.O. Box: 2713, Doha, Qatar, Tel: 0097444034783; E-mail: Hassan.Aziz@qu.edu.qa

Received August 03, 2016; Accepted September 13, 2016; Published September 23, 2016

Citation: Aziz HA, Guled A (2016) Cloud Computing and Healthcare Services. J Biosens Bioelectron 7: 220. doi: 10.4172/2155-6210.1000220

Copyright: ( 2016 Aziz HA, et al. This is an open-access article distributed under the terms of the Creative Commons Attribution License, which permits unrestricted use, distribution, and reproduction in any medium, provided the original author and source are credited. 
to cloud - based healthcare services changes the traditional current concept and takes it to another whole level, where it breaks the physical boundaries of healthcare organizations and promotes a new era of self and domestic care. An example of this is "centricity practice" a cloud based healthcare service by General Electric (GE) that is focused on Patient Self-Service Portal that helped patients communicate with their healthcare providers remotely and securely [4]. Dell also released "Practice Fusion" which offered an EHR package for small to medium medical enterprises [5].

Healthcare organizations in the US are driven by Health Information Technology for Economic and Clinical Health (HITECH) Act. The act supports the move of medical practices and healthcare organization from the traditional paper-based systems to cloud-based systems. This movement falls under the meaningful use which is encouraged and supported by President Obama administration through the establishment of Medicare and Medicaid programs under the HITEC Act. In the UK, the National Health Service (NHS) took big steps to allow patient's access to their own EHRs. It was due to a proposal made in 2011 by the NHS Future Forum [6] that focused on revolutionizing healthcare practices and involving patients in the decision making process and to take control over their own health. In addition, the proposal emphasized on the financial benefits of using cloud-based healthcare systems where less number of resources would be spent to accommodate patients multiple visits when it could be done simply and remotely by just a few computer clicks [7].

\section{Security and privacy of healthcare data with cloud computing}

Considering the advantages resulting from using cloud-based healthcare systems, there are always concerns regarding security and privacy. In order to maintain the security and privacy of healthcare data, both cloud service providers and healthcare organizations should take extreme measures to secure a safe handling of patient's data. Most of the concerns are perceived from the concept of having medical data and information that are classified as confidential to be in cloud servers, a virtual world where information can be easily hacked. Therefore, high security measures and guarantees should be enforced before deploying any cloud-based healthcare services. Governments' rules and regulations should be in place to ensure that cloud service providers should comply with the legislation and apply all necessary means to protect patients' data security and privacy. The Health Insurance Portability and Accountability Act (HIPAA) oversees and regulates privacy and security of patients' data whether cloud systems were involved or not. HIPAA formulated a privacy rule identified as Protected Health Information (PHI) that ensures patient's information can't be used without a court order or patient's consent and authorization.

HIPAA security rules addressed issues concerning the digitalization of healthcare data, in addition to the responsibility of healthcare organizations to securely handle this process. Moreover, EHR systems come with a built- in security measures to ensure the availability of a safe environment for the management of patients' data. Patients are part of this process of securely handling their health data and information. They need to be aware of the presence of a third party involvement in the management of their health data which is the cloud server provider. It is the responsibility of healthcare organizations to communicate legislation issues involved with using cloud-based healthcare services with their patients. In addition, to sustain the concept of a meaningful use, healthcare providers need to educate patients about their role in this process and how they can access their data in a secure manner. Within healthcare organization, a role-based access system should be kept in place to specify what kind of access level healthcare professionals will have according to their job specifications.

Proper authentication systems added to the role-based system supports more secure environment to store and manage patients' data. In the process of migrating healthcare data to a cloud system, there are security precautions to be taken which include data encryption [8]. While data encryption seems be the most secure method for handling patients data, however not all EHR systems are compatible with standard encryption methods. This is due to the multiple users who each have a role-based access, nevertheless their access might overlap because of their job specialty which requires different route access to the EHR. This exemplifies the need to develop more advanced encryption methods that are suitable for the EHR. An example is using a Symmetric-Key Encryption (SKE) [9]. It is an advanced encryption technique which is considered efficient; however it still introduces complexity in EHR systems. This is due to the encryption technique that requires all healthcare providers to use one key for encryption and decryption. What is risky about this method is that in case there was a breach in the encryption all EHR data will be compromised at the same time. Another example of existing encryption techniques is using a Public-Key Encryption (PKE) [9]. It is an expensive technique where a public-key infrastructure (PKI) is used to maintain and manage the encryption. Therefore, it is not practical for EHR storage and other suitable techniques should be considered. Ciphertext Policy AttributeBased Encryption (CP-ABE) has been proposed as much suitable and better fit for healthcare organizations [9]. It is an encryption method that provides effective solutions to some of the issues related to standard encryption mechanisms. Also it utilizes user roles for accessing and handling secure data. It provides reasonable performance and consumes small storage area; therefore it can be used as a replacement to standard encryption mechanisms in cloud-based EHR systems [9].

Other means of safety measures include monitoring of system access, where logs are created to register all individuals who access the system. This is beneficial in investigating cases of systems breach. Cloud network security is a must for a secure transfer of data. Digital signature is another method that helps in the process of data security, in addition to having a backup server for the data saved in a cloud as a security measure in case the server is hacked or damaged [8].

\section{Examples of cloud computing applications used in healthcare management emergency healthcare process using cloud service}

Emergency healthcare processes involve both pre-hospital and in-hospital services. There are varieties of interrelated activities from the administrative to the medical care. Therefore, proper means of information exchange and communication is needed between healthcare organizations and Emergency Medical Services (EMS). The availability of patients' information affects the care plan for emergency cases, also it influences the transportation means (e.g. helicopter or ambulance) and proper relaying of patient's condition. Moreover, it leads to a more accurate case prioritizing based on the severity, avoidance of unnecessary testing and overall improvement of emergency care quality. Since information exchange is important between healthcare organizations and EMS, likewise emergency responders' communication is as important. That is why the Organization for the Advancement of Structured Information Standards (OASIS) Emergency Management Technical Committee, designed the Emergency Data Exchange Language (EDXL) to help EMS share valuable information across regional and national organizations. Having cloud computing systems integrated with emergency care services systems facilitate a quicker method of data retrieval from patient's personal healthcare 
record (PHR) or EHR. This information will provide guidance to the paramedics to make the right decision and manage patients' condition based on their medical history.

Furthermore, emergency personal healthcare record (EPHR) is formed to help paramedics access needed information that are continuously updated from various sources. This information is classified into: emergency care oriented documents, patient oriented documents which include the patient medical history, and the resource oriented documents which are educational resources. EPHR aids EMS personnel to access patient-oriented information that can be provided in either Health Level 7 (HL7), or the Clinical Document Architecture (CDA) standard through the usage of cloud computing services [10].

\section{Cloud computing and body sensors}

Body Sensor Networks (BSN) is a part of the Wireless Sensor Networks (WSN). It is composed of sensor nodes used to monitor physical, environmental, or human conditions. BSN is used in the medical field where it remotely transmits signals to monitor changes in patients' condition e.g. temperature, heart rate, and blood pressure. These sensors provide large amounts of real-time data that can be sent and stored in cloud servers and transferred to healthcare organizations where data are analyzed and interpreted by medical staff. Cloud computing offers the means to utilize heterogeneous sensors, data storage, analysis and sharing results with EHRs.

Body cloud system is a merger between both the BSN and cloud computing that allows data collection and transfer to healthcare organizations. The way sensors function is either that they are attached to the body surface or implanted in it without impairing patients' every day normal activities. Real-time data are collected from these sensors, and then transmitted wirelessly to healthcare external processing units where patient's physician monitor and analyse those data. An example of BSN is the auto-injecting of insulin through a pump when there is a drop in the insulin levels. Another is the detection of a heart attack episode by checking the change in the heart waves and vital signs. BSN applications are non-invasive and considerably cost effective. They provide real-time data with an aim to improve the quality of healthcare services in the long run by early detection or prevention of diseases [11].

Advanced mobile applications combined with cloud computing technology assist healthcare professionals to use such systems. These systems are more beneficial for those patients with chronic conditions who need continuous monitoring and are struggling to seek consultation from healthcare clinics/hospitals like patients residing in rural areas. Cloud computing has the characteristic of elasticity which is the ability of a system to expand and contract depending on the clients usage for the system. Therefore, cloud computing resolves challenges that are related to scalability and feasibility. Cloud infrastructure is characterized with a high throughput and high volume storage, two important factors for efficient data analysis of large patients' population [12]. Figure 1 shows an example of how mobile devices and WBANs are connected through the internet where real-time data are sent to cloud servers (Figure 1).

\section{Digital imaging communication in medicine and cloud com- puting}

Picture archiving and communication system (PACS) is a tool used in the clinical settings for handling medical images. It is used to store, retrieve and distribute medical images that are critical to support medical decisions and complex treatment procedures. Digital Imaging Communication in Medicine (DICOM) is the standard that supports storage format and distribution of medical images. However, the usage of DICOM is limited within organizational Virtual Private Network (VPN) connections. Web 2.0 is partially compatible with DICOM and it permits only images retrieval over the Web. These limitations prevents medical staff from outside the organization to access the PACS archive [13]. Medical institutions rely on manual processes including VPN, which require point-to-point configurations and is not scalable. Other ways include through CD/DVD or email. However, these methods are not efficient when there is a need to access medical imaging remotely for an emergency situation [14]. Using systems where cloud computing service integrates with DICOM solves such problems. It facilitates access to medical images online directly from the EHR or PHR with the use of mobile device. As it is illustrated in Figure 2, when patients want to access their own health records that are stored in cloud they connect to it through their mobile phones. There are imaging module that can be installed in mobile phones that can help display medical images by decoding them in DICOM format [15-17] (Figure 2).

\section{Conclusion}

Cloud computing system is a measured service that has the ability to automatically control and optimize resource usage in regards to the type of service provided. Healthcare organizations needs innovative and cost-effective methods to assist healthcare providers find more productive ways to address growing numbers of patients' data Electronic Health records (EHR) paired with cloud computing systems brings a solution for the "big data" challenge where it accommodates storage resources and facilitates the process of sharing patients' data between healthcare providers.

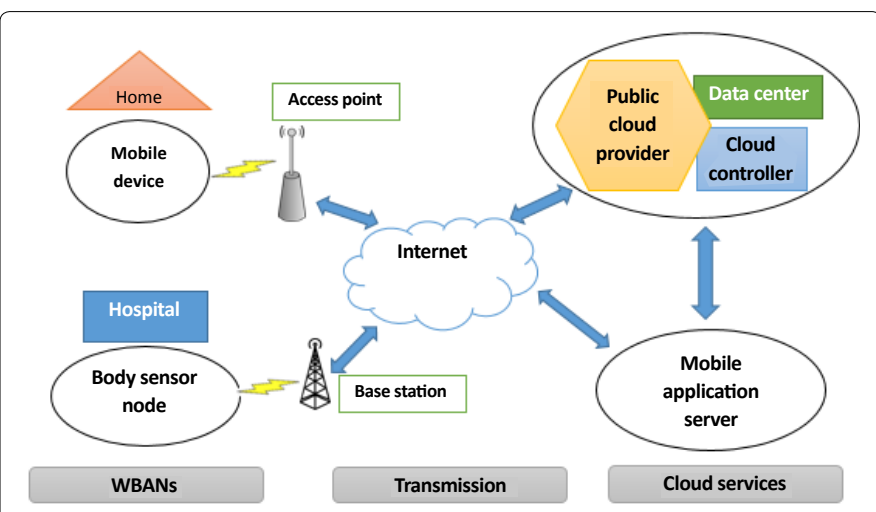

Figure 1: WBANs with mobile cloud computing [16]

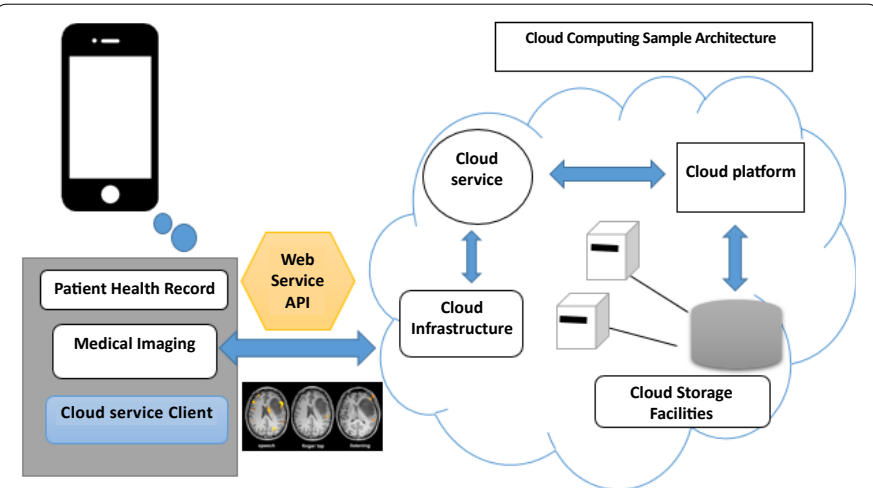

Figure 2: Illustration of cloud computing usage in medical image display [17] 


\section{References}

1. Mell P, Grance T (2011) The NIST definition of cloud computing

2. Aziz HA (2015) Health Informatics - Introduction. Clin Lab Sci 28: 238-239

3. Fabian B, Ermakova T, Junghanns P (2015) Collaborative and secure sharing of healthcare data in multi-clouds. Information Systems 48: 132-150.

4. GE Healthcare (2010) Centricity practice solution going beyond meaningful use.

5. Kolakowski N (2010) Dell, practice fusion to offer medical records system.

6. Barrow M (2011) Patients get right to see medical records online. The Times.

7. Sultan N (2014) Making use of Cloud computing for healthcare provision: Opportunities and challenges. Int J Inform Manage 34: 177-184.

8. Rodrigues JJ, de la Torre I, Fernández G, López-Coronado M (2013) Analysis of the security and privacy requirements of cloud-based electronic health records systems. J Med Internet Res 15: e186.

9. Alshehri S, Radziszowski SP, Raj RK (2012) Secure access for healthcare data in the cloud using ciphertext-policy attribute-based encryption. Data Engineering Workshops (ICDEW), 2012 IEEE 28th International Conference. pp: 143-146.

10. Fortino G, Pathan M, Di Fatta G (2012) BodyCloud: Integration of Cloud Computing and body sensor networks. Cloud Computing Technology and Science (CloudCom), 2012 IEEE 4th International Conference. pp 851-856.

11. Poulymenopoulou M, Malamateniou F, Vassilacopoulos G (2011) Emergency Healthcare process automation using mobile computing and cloud services. J Med Syst 36: 3233-3241.

12. Pandey S, Voorsluys W, Niu S, Khandoker A, Buyya R (2012) An autonomic Cloud environment for hosting ECG data analysis services. Future Generation Computer Systems 28: 147-154.

13. Aziz H, Madani A (2015) Evolution of the web and its uses in healthcare. Clin Lab Sci 28: 245-249.

14. Wan J, Zou C, Ullah S, Lai CF, Zhou M, et al. (2013) Cloud-enabled wireless body area networks for pervasive healthcare. Network IEEE 27: 56-61.

15. Doukas C, Pliakas T, Maglogiannis I (2010) Mobile healthcare information management utilizing Cloud Computing and Android OS. Engineering in Medicine and Biology Society (EMBC), 2010 Annual International Conference of the IEEE. pp: 1037-1040.

16. Silva LAB, Costa C, Oliveira JL (2013) DICOM relay over the cloud. Int J Comput Assist Radiol Surg 8: 323-333.

17. Doukas C, Pliakas T, Maglogiannis I (2010) Mobile healthcare information management utilizing Cloud Computing and Android OS. Engineering in Medicine and Biology Society (EMBC), 2010 Annual International Conference of the IEEE. pp: 1037-1040. 